\title{
Development of an on-board failure diagnostics and prognostics system for Solid Rocket Booster
}

\author{
Vadim N. Smelyanskiy \\ NASA Ames Research Center, Mail Stop 269-2, Moffett Field, CA 94035, USA \\ Dmitry G. Luchinsky, Vyatcheslav V. Osipov \\ NASA Ames Research Center, Mail Stop 269-2, Moffett Field, CA 94035, USA and \\ Mission Critical Technologies Inc., 2041Rosecrans Ave. Suite 225 El Segundo, CA 90245 \\ Dogan A. Timucin, Serdar Uckun \\ NASA Ames Research Center, Mail Stop 269-2, Moffett Field, CA 94035, USA
}

\begin{abstract}
We develop a case breach model for the on-board fault diagnostics and prognostics system for subscale solid-rocket boosters (SRBs). The model development was motivated by recent ground firing tests, in which a deviation of measured time-traces from the predicted time-series was observed. A modified model takes into account the nozzle ablation, including the effect of roughness of the nozzle surface, the geometry of the fault, and erosion and burning of the walls of the hole in the metal case. The derived low-dimensional performance model (LDPM) of the fault can reproduce the observed time-series data very well. To verify the performance of the LDPM we build a FLUENT model of the case breach fault and demonstrate a good agreement between theoretical predictions based on the analytical solution of the model equations and the results of the FLUENT simulations. We then incorporate the derived LDPM into an inferential Bayesian framework and verify performance of the Bayesian algorithm for the diagnostics and prognostics of the case breach fault. It is shown that the obtained LDPM allows one to track parameters of the SRB during the flight in real time, to diagnose case breach fault, and to predict its values in the future. The application of the method to fault diagnostics and prognostics (FD\&P) of other SRB faults modes is discussed.
\end{abstract}

\section{Introduction}

Development of on-board FD\&P system for the Solid Rocket Boosters is a NASA requirement for the next-generation Crew Exploration and Heavy-Lift Launch Vehicles. The limited number of sensors (typically head pressure, temperature and acceleration) and short time window between the detectable onset of a variety of catastrophic failures and a crew abort (typically a few seconds) dictate a model based approach to development of the FD\&P system for SRBs. Such a system should accommodate the abrupt changes of the model parameters in various nonlinear dynamical off-nominal regimes. The later objective can only be achieved by incorporating expert knowledge about characteristic dynamical features of various off-nominal regimes and by utilizing deep physical understanding of the underlying physical processes. Examples of the most probable faults in SRBs include: (i) combustion instabilities ${ }^{1-3}$, (ii) bore chocking ${ }^{4-6}$, and (iii) case breach ${ }^{7-9}$. Clearly the development of the FD\&P that incorporates physics models of the faults has to verified and validated in a multi-stage procedure involving highfidelity modeling, ground and flying tests. In our earlier work we have developed a novel Bayesian framework $^{7,10-13}$ that can infer parameters of nonlinear stochastic dynamical models. We have also derived a low-dimensional performance model (LDPM) of the SRBs ${ }^{7,12,13}$ and demonstrated that our Bayesian framework can infer and track parameters of the LDPM in real time. Specifically, the LDPM 
of the case breach fault was developed in ${ }^{7}$ in anticipation of the ground firing tests. The results of the tests (the discussion will be provided elsewhere) have demonstrated however, that further details have to be included into the model to describe the observed fault dynamics.

In this paper we develop a detailed model of the case breach fault that accurately reproduces characteristic dynamical features of the fault observed in the ground tests and incorporate it into the Bayesian inferential framework. The essential modifications of the model include (i) the dynamics of the nozzle ablation; (ii) a given propellant geometry and the relation between burn distance and burning area; (iii) the dynamics of melting, ablation, and burning of the metal walls in the hole through the metal case; (iv) the dynamics of silicon melting in the nozzle walls; and (v) the geometry of the fault. These modifications are also included into a high-fidelity model of the case breach fault built in FLUENT. Using the results of the theoretical analysis of the case breach fault and FLUENT simulations we derive and verify a corresponding LDPM and incorporate it into a Bayesian inferential framework as a part of on-board FD\&P system for SRBs. We use synthetic data generated by the LDPM to verify the accuracy and the time resolution of the diagnostics and prognostics of the case breach fault. Finally we discuss the possibility of extension of this approach to encompass other SRBs faults.

\section{NOMENCLATURE}

$\begin{array}{ll}\rho & =\text { gas density } \\ p & =\text { gas pressure } \\ T & =\text { gas temperature } \\ u & =\text { gas velocity } \\ v_{m} & =\text { velocity of metal melting front } \\ v_{n} & =\text { velocity of nozzle ablation front } \\ c & =\text { sound velocity } \\ c_{0} & =\text { sound velocity at stagnation point } \\ M & =\text { Mach number, } M=u / c \\ M_{0} & =\text { Mach number, } M_{0}=u / c_{0} \\ c_{V} & =\text { specific heat for the constant volume } \\ c_{P} & =\text { specific heat for the constant pressure } \\ \gamma & =\text { ratio of specific heats } \gamma=c_{P} / c_{V} \\ l & =\text { perimeter of propellant cross-section } \\ l_{h} & =\text { perimeter of hole cross-section } \\ l_{r} & =\text { surface roughness } \\ r_{e} & =\text { radius of nozzle exit } \\ r_{h} & =\text { radius of leak hole } \\ s_{h} & =\text { cross-section of the hole throat } \\ r_{t} & =\text { radius of the nozzle throat } \\ s_{t} & =\text { cross section of the nozzle throat } \\ L & =\text { length of the propellant grain } \\ L_{0} & =\text { characteristic length scale } \\ S_{b} & =\text { total area of the burning surface } \\ F_{N} & =\text { normal thrust } \\ F_{h} & =\text { additional thrust produced by hole gas flow } \\ r_{b} & =\text { burning rate of solid propellant } \\ n & =\text { exponent for burning rate of the propellant } \\ n & \end{array}$




$\begin{array}{ll}R & =\text { burn distance of the propellant } \\ \rho_{p} & =\text { density of the solid propellant } \\ H_{p} & =\text { combustion heat of the solid propellant } \\ Q & =\text { heat flow from the gas to the walls of the hole } \\ S & =\text { cross-section of the combustion chamber } \\ f_{t r} & =\text { surface friction force } \\ T_{m} & =\text { melting temperature point of metal } \\ T_{a} & =\text { critical temperature of the nozzle ablation } \\ T_{c} & =\text { temperature of metal case far from hole } \\ c_{m} & =\text { specific heat of case metal } \\ q_{i n s} & =\text { latent heat of insulator ablation } \\ q_{m} & =\text { latent heat of metal melting } \\ c_{n} & =\text { specific heat of nozzle material } \\ q_{n} & =\text { specific heat of ablation of insulator layer } \\ \rho_{m} & =\text { density of case metal } \\ \rho_{n} & =\text { density of nozzle material } \\ k & =\text { the thermal conductivity of metal } \\ \mu & =\text { dynamical viscosity of hot gas } \\ \operatorname{Pr} & =\text { the Prandtl number, Pr= } \mu C_{p} / k \\ h & =\text { subscript for gas parameters in the hole } \\ t & =\text { subscript for gas parameters at the nozzle throat } \\ N & =\text { subscript for parameters in normal regime } \\ O & =\text { subscript for stagnation values of gas parameters }\end{array}$

\section{HIGH-FIDELITY MODEL OF THE CASE BREACH}

\section{A. Basic model}

For SRBs with high length-to-diameter ratios a very good approximation to the internal ballistics can be obtained using a modified model by Salita ${ }^{15}$ (see also Sorkin ${ }^{16}$ )

$$
\begin{aligned}
& \partial_{t}(S \rho)=-\partial_{x}(S \rho u)+\rho_{p} r_{b} l+\xi_{1}(t), \\
& \partial_{t}\left[S \rho\left(c_{V} T+u^{2} / 2\right)\right]=-\partial_{x}\left[S \rho u\left(c_{P} T+u^{2} / 2\right)\right]+H \rho_{p} r_{b} l+\xi_{2}(t), \\
& \partial_{t}(S \rho u)=-\partial_{x}\left(S \rho u^{2}\right)-S \partial_{x} p-\alpha \rho u^{2} l(x)+\xi_{3}(t) .
\end{aligned}
$$

Here we have taken into account small fluctuations in gas dynamics that arise mainly due to the propellant density variations and external white Gaussian noise $\xi_{i}(t)$

$$
\left\langle\xi_{i}(t)\right\rangle=0, \quad\left\langle\xi_{i}(t) \xi_{j}(0)\right\rangle=\sqrt{\sigma} \delta(t) \delta_{i j} .
$$

These equations for continuity of the mass, momentum and energy of the gas flow in the combustion chamber have to be extended by including equations for dynamics of the burn distance, nozzle ablation, and erosion of the metal walls. In the simplest case of the propellant burning law and Bartz' approximation for the heat transfer between the gas flow and walls of the nozzle and the hole in the metal case we have the following equations for the burn distance (in what follows we use dot to denote time derivative)

$$
\dot{R}=r_{b}(p)=r_{c}\left(\frac{p}{p_{c}}\right)^{n}=a p^{n}
$$


nozzle throat ablation

$$
\dot{r}_{t}=v_{t}\left(\frac{\rho v}{(\rho v)_{t}}\right)^{1-\beta}\left(\frac{r_{t}(t, x)}{r_{t 0}}\right)^{-\beta},
$$

and erosion of the metal walls of the hole in the rocket case

$$
\dot{r}_{h}=v_{a b l}=v_{m}\left(\frac{\rho v}{(\rho v)_{h t}}\right)^{1-\beta}\left(\frac{r_{h}(t, x)}{r_{h 0}}\right)^{-\beta} .
$$

(see the Appendix for the derivation of these equations). It is assumed that the equation of state for an ideal gas holds in the combustion chamber

$$
\frac{p}{\rho}=\left(C_{p}-C_{V}\right) T=\frac{p_{0}}{\rho_{0}}\left(\frac{T}{T_{0}}\right)=\frac{c_{0}^{2}}{\gamma}\left(\frac{T}{T_{0}}\right)
$$

To incorporate real propellant geometry the following key assumption is introduced (cf Ref. 9): at every moment of time the burning area $S_{b}$ is determined by the burn distance $R_{p}$ and the corresponding design curve

$$
S_{b}=f\left(R_{p}\right)
$$

The system (1)-(7) is completed by adding equation for the nozzle exit ablation

$$
\dot{r}_{e}=v_{e}\left(\frac{\rho v}{(\rho v)_{e t}}\right)^{1-\beta}\left(\frac{r_{e}(t, x)}{r_{e}}\right)^{-\beta}\left(\frac{T-T_{a}}{T_{*}-T_{a}}\right)
$$

and equation for the nozzle $F_{N}$ and fault-induced $F_{h}$ thrusts

$$
\begin{aligned}
F_{N} & =\left[(\rho u)_{t} u_{t, e x}+\left(p_{t, e x}-p_{a}\right)\right] s_{t}, \\
F_{h} & =\left[(\rho u)_{h} u_{h, e x}+\left(p_{h, e x}-p_{a}\right)\right] s_{h} .
\end{aligned}
$$

Equations (1)-(10) represent the basic model of the case breach fault that incorporates the essential dynamical features of the fault-induced changes in the internal ballistics of the gas flow. In the following sections we will validate this model using results of the FLUENT simulations. Next we will extend this model to include experimentally observed dynamics of the metal erosion and nozzle ablation. Then we will derive the corresponding LDPM and incorporate it into the Bayesian inferential framework.
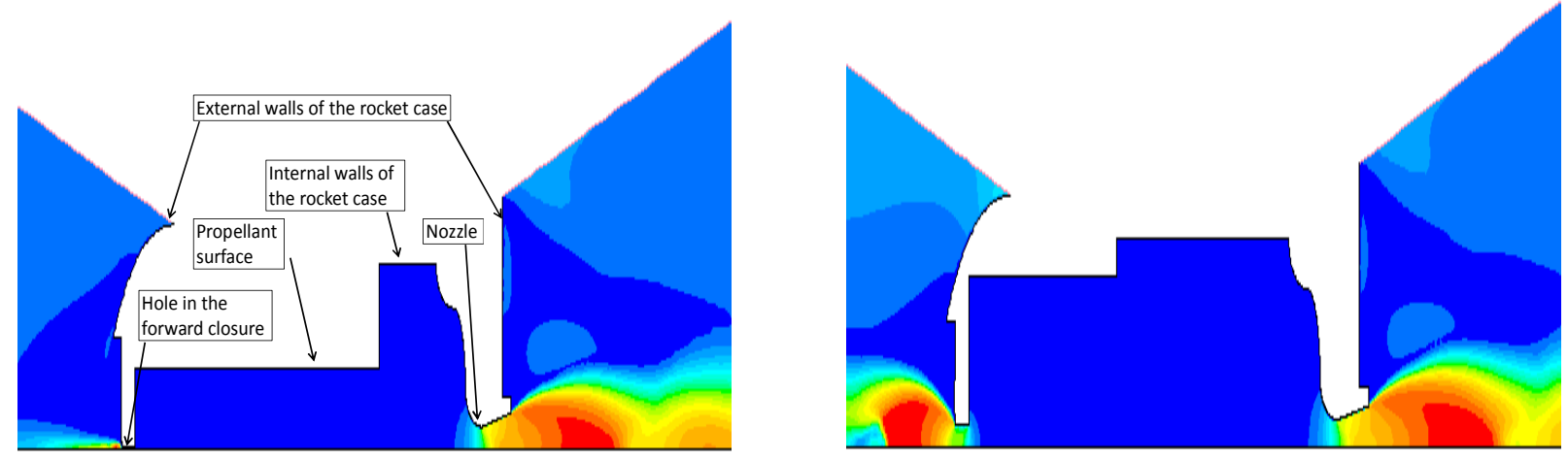

Figure 1 (left) Velocity distribution obtained using FLUENT simulations after 0.14 sec. The geometry of the model surfaces is shown in the figure. Note that the hole wall, propellant surface wall, and the nozzle wall are deforming according to the equations (3)-(5), (8). (right) Velocity distribution generated by the FLUENT model for $t=5.64 \mathrm{sec}$. Note the changes in the geometry of the rocket walls and the corresponding changes in the velocity distribution. 

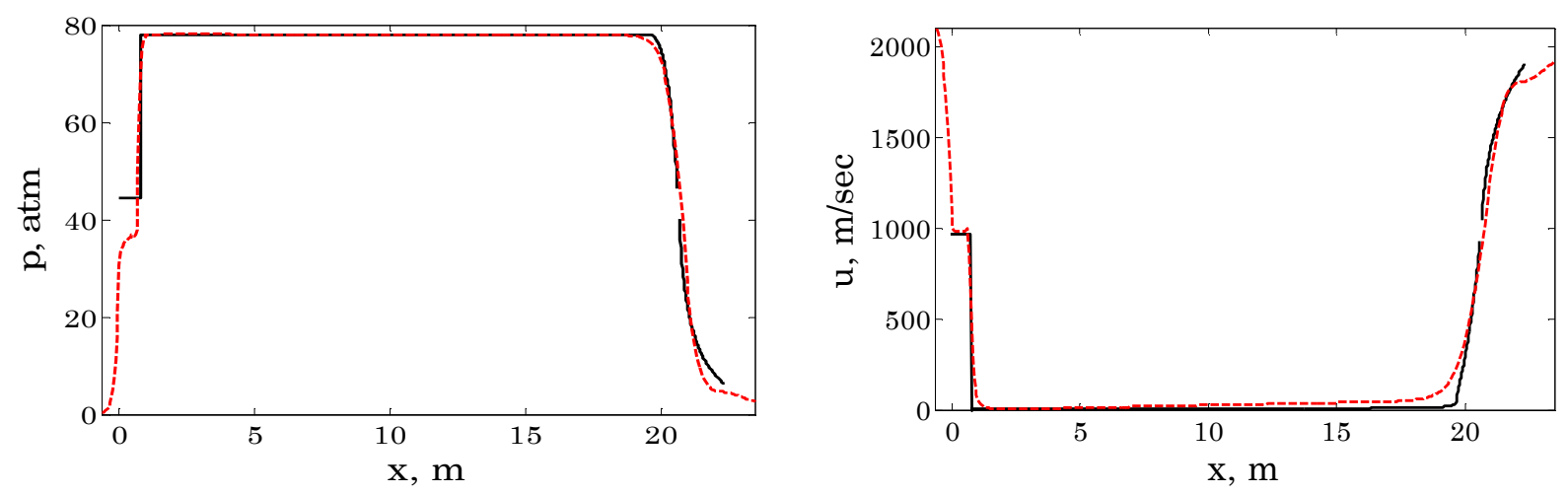

Figure 2 Axial velocity (left) and pressure (right) profiles generated by the FLUENT model for $t=0.05$ sec (black solid line) as compared to the analytical solutions (red dashed lines) given by the Eqs (11)-(13).

\section{B. Validation of the basic model using FLUENT simulations}

To validate the model of the case breach fault (1)-(10) introduced above we build a FLUENT model of the case breach (see Figure 1). Next we notice that of the propellant surface, metal erosion, and nozzle ablation are relatively slow processes as compared to the time scale for redistribution of gas parameters along the combustion chamber (typically $t_{r e l} \approx L / c \sim 10 \mathrm{msec}$ ). Under these conditions it becomes possible to find stationary solutions of the Eqs (1) analytically in the combustion chamber

$$
\rho=\rho_{0}\left(1+\frac{\gamma+1}{2} \frac{u^{2}}{c_{0}^{2}}\right)^{-1}, p=p_{0}\left(1+\frac{\gamma+1}{2} \frac{u^{2}}{c_{0}^{2}}\right)^{-1}\left(1-\frac{\gamma-1}{2} \frac{u^{2}}{c_{0}^{2}}\right)^{-1}, v=u_{L} \frac{x}{L}\left(1+\frac{3(\gamma+1)-2 n \gamma)}{6 c_{0}^{2}} u_{L}^{2} \frac{x^{2}}{L^{2}}\right)
$$

and in the nozzle area

$$
p=p_{0}\left(1-\frac{(\gamma-1)}{2} M_{0}^{2}\right)^{\frac{\gamma}{\gamma-1}}, \rho=\rho_{0}\left(1-\frac{(\gamma-1)}{2} M_{0}^{2}\right)^{\frac{1}{\gamma-1}}, T=T_{0}\left(1-\frac{(\gamma-1)}{2} M_{0}^{2}\right),
$$

where $M_{0}$ is given by the solution of the nozzle equation

$$
\mathrm{M}_{0}\left(1-\frac{(\gamma-1)}{2} M_{0}^{2}\right)^{\frac{1}{\gamma-1}}=\frac{s_{t}}{\Gamma S}
$$

The results of the comparison of the analytical distributions obtained using Eqs (11)-(13) with the axial velocity and pressure distributions obtained using FLUENT simulations are shown in the Figure 2 . It can be seen from the figure that the model (1)-(10) provides a very good approximation to the results of FLUENT simulations. Note that the difference in the time scales for dynamics of burn distance, metal erosion, and nozzle ablation as compared to the characteristic relaxation time of the distributions to their quasi-stationary values $t_{r e l}$, allows us to integrate equations (1)-(13) in quasi-stationary approximation. As a result we obtain analytical solution for the quasi-stationary dynamics of the axial distributions of the gas parameters in the combustion chamber and in the nozzle area. The comparison of this analytical solution with the results of FLUENT simulations also demonstrates good agreement between the theory and numerical solution of the high-fidelity model.

A further validation and verification of the case breach model (1)-(10) can be obtained using results of the ground firing test, as will be described in detail elsewhere. Here we will discuss necessary modifications of the basic case breach model that must be introduced to explain the experimentally observed deviations of the case breach dynamics from the predictions based on the model (1)-(10). 


\section{MODIFICATIONS OF THE MODEL FOR METAL EROSION AND NOZZLE ABLATION}

Although the basic model (1)-(10) introduced above provides deep insight into the dynamics of the case breach fault, for a given propellant geometry and given design curve $S_{b}=f\left(R_{p}\right)$, experimental results obtained during the ground firing tests suggest that more details must be added to the model to reproduce experimentally observed time-traces. In particular, the experimental results demonstrate some deviations in the nozzle ablation and metal erosion rates from that given by Eqs (4), (5), (8). These deviations are related mainly to the complex geometry of the actual fault in the forward closure, to the heating of the metal case during erosion of the metal walls, and to the erosion of the nozzle wall surface during ablation process that changes the rate of ablation. Some other physical effects such as thermal expansion of the materials and wave formations on the walls of the nozzle throat will be considered in more detail elsewhere.

The study shows that melting and burning of metal surface (a result of reactions with oxidizing agents of combustion products) play an essential role in the hole growth dynamics. To consider these effects we assume that

$$
\dot{r}_{h}=v_{f}+v_{b}
$$

where $v_{f}$ and $v_{b}$ are velocities of melting and burning of metal surface (see Appendix).

To take into account the effect of the surface roughness on the nozzle ablation the model of the ablation velocity is modified as follows (see Appendix)

$$
\dot{r}_{t}=\eta_{r} v_{m}\left(\frac{p_{0}}{p_{\max }}\right)^{1-\beta}\left(\frac{r_{t}}{r_{t 0}}\right)^{-\beta}
$$

where $r_{t 0}$ is the initial value of the nozzle throat radius and

$$
\eta_{r}=\frac{a l_{r}+\varepsilon R_{n t o}}{l_{r}+\varepsilon R_{n t o}}, \quad i_{r}=v_{m}\left(\frac{p_{0}}{p_{\max }}\right)^{1-\beta}\left(\frac{r_{t}}{r_{t 0}}\right)^{-\beta}
$$

Here $\eta_{r}$ is a coefficient describing an effect of the surface roughness, $a_{r}$ and $\varepsilon$ are fitting parameters.

In practice, the dynamics of the fault strongly depends on its geometry. For example we consider a fault in the closure of the pressure sensor, in the form of a metal disk of radius $R_{d}$ (see Figure 3 ). This disk is covered by insulator material. We assume that a small leak arises on the disk boundary at moment $t=t_{0}$. The experiments show that the insulator layer is quickly ablated by the powerful hot gas flow streaming

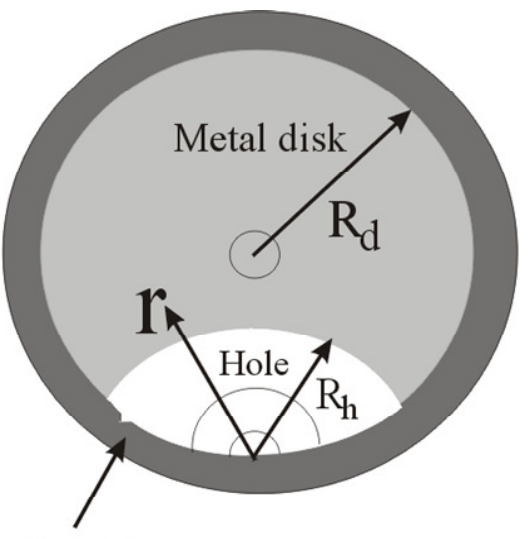

Insulator

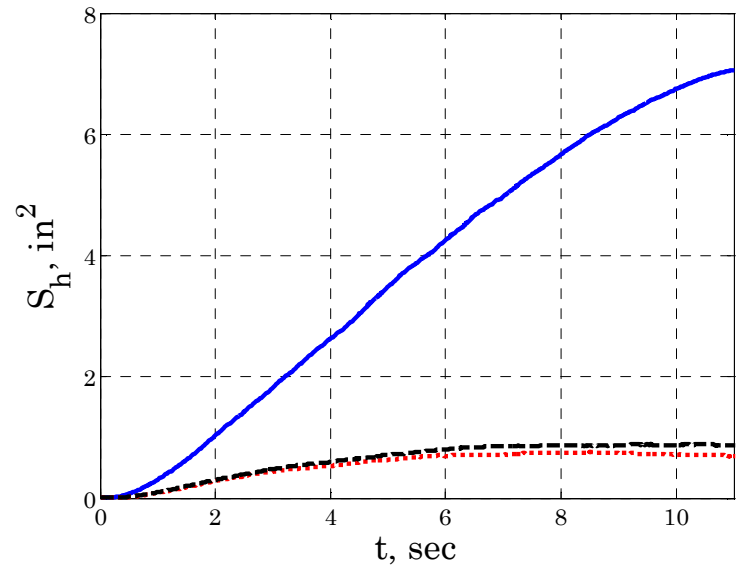

Figure 3 (left) Sketch of the fault geometry. (right) Dynamics of the hole growth that takes into account radiation, and erosion (blue solid line) as compared to the growth dynamics that neglects erosion (black dashed line) and growth dynamics neglecting both erosion and radiation (red dotted line). Note the nonlinear regime of the fault 
through a leak hole. Therefore, the small hole grows due to melting and/or burning of the metal surface under the action of the hot gas flow effluent from the combustion chamber. In the simplest case the hole is in the middle of the metal disk and its cross-section area is given by $s_{h}=\pi r_{h}^{2}$. If on the other hand the hole appears on the perimeter of the disk it can be shown that its cross-section is given by

$$
s_{h}=\pi R_{d}^{2}-\left(2 R_{d}^{2}-r_{h}^{2}\right) \arccos \left(\frac{r_{h}}{2 R_{d}}\right)-\frac{r_{h}}{2} \sqrt{4 R_{d}^{2}-r_{h}^{2}}
$$

Therefore, it can be seen that in general hole dynamics can be a complex function of time, which can be measured, e.g. as a polynomial function, which coefficients are free fitting model parameters.

\section{LOW-DIMENSIONAL MODEL OF THE CASE BREACH FAULT}

To derive the LDPM of the case breach fault we notice that for a subscale motors the flow in the chamber can be modeled very accurately using a zero-dimensional approximation ${ }^{15,18,19}$. Therefore the LDPM of the case breach fault in the sub-scale motor can be derived by integration of Eqs (1) along the rocket axis and by adding Eqs (3)-(5), (7) to obtain

$$
\begin{aligned}
& \dot{\rho}=-\frac{c_{0} \Gamma s_{e t}}{V r_{b}} \rho \sqrt{\frac{p}{\rho}}+\frac{S_{b}}{V}\left(\rho_{p}-\rho\right) p^{n}+a_{1} \xi_{1}(t), \\
& \dot{p}=-\frac{c_{0} \gamma \Gamma s_{e t}}{V r_{b}} p \sqrt{\frac{p}{\rho}}+\frac{S_{b}}{V}\left(\gamma \rho_{p}-p\right) p^{n}+a_{2} \xi_{2}(t), \\
& \dot{R}=a p^{n}, \quad \dot{V}=S_{b} \dot{R}=S_{b} a p^{n}, \\
& s_{e t}=\pi r_{t}^{2}+s_{h}, \quad S_{b}=f(R), \\
& F_{N}=\left[(\rho u)_{t} u_{t, e x}+\left(p_{t, e x}-p_{a}\right)\right] s_{t}, \\
& F_{h}=\left[(\rho u)_{h} u_{h, e x}+\left(p_{h, e x}-p_{a}\right)\right] s_{h} .
\end{aligned}
$$

Here the following dimensionless variables are used

$$
p \rightarrow \frac{p_{0}}{p_{m}}, \rho \rightarrow \frac{\rho_{0}}{\rho_{m}} ; t \rightarrow \frac{t r_{b}\left(p_{m}\right)}{L_{0}}, r_{t} \rightarrow \frac{r_{t}}{L_{0}}, F \rightarrow \frac{F}{L_{0}^{2}}, V \rightarrow \frac{V}{L_{0}^{3}}, r_{h t} \rightarrow \frac{r_{h t}}{L_{0}}, R \rightarrow \frac{R}{L_{0}}, s_{e t} \rightarrow \frac{s_{e t}}{L_{0}^{2}},
$$

where subscript $m$ refers to the maximum reference values of the pressure and density. This set of equations together with Eqs. (14)-(17) represent the LDPM of the case breach derived in this paper.

Important novel features of the case breach LDPM derived above are the following:

- $\quad$ The burning area of the propellant $S_{b}$ is calculated using a given design curve $S_{b}=f(R)$;

- The dynamics of the metal erosion and nozzle ablation is taken into account;

- The dynamics of the volume of the combustion chamber is taken into account in Eqs. (18).

With these modifications the LDPM (18) can very accurately reproduce the results of the ground tests. Typical time-series data for the case breach fault obtained using (18) are shown in Figure 4. In these figures the fault occurs at $t_{f}=0.5 \mathrm{sec}$ and the metal erosion rate in the regime of constant erosion is 0.15 in/sec. Importantly, the derived model can reproduce very accurately the experimental time-traces of internal ballistics observed in the ground tests, as will be described in details elsewhere. It allows us to incorporate obtained an LDPM into the Bayesian inferential framework of the on-board FD\&P of SRBs 

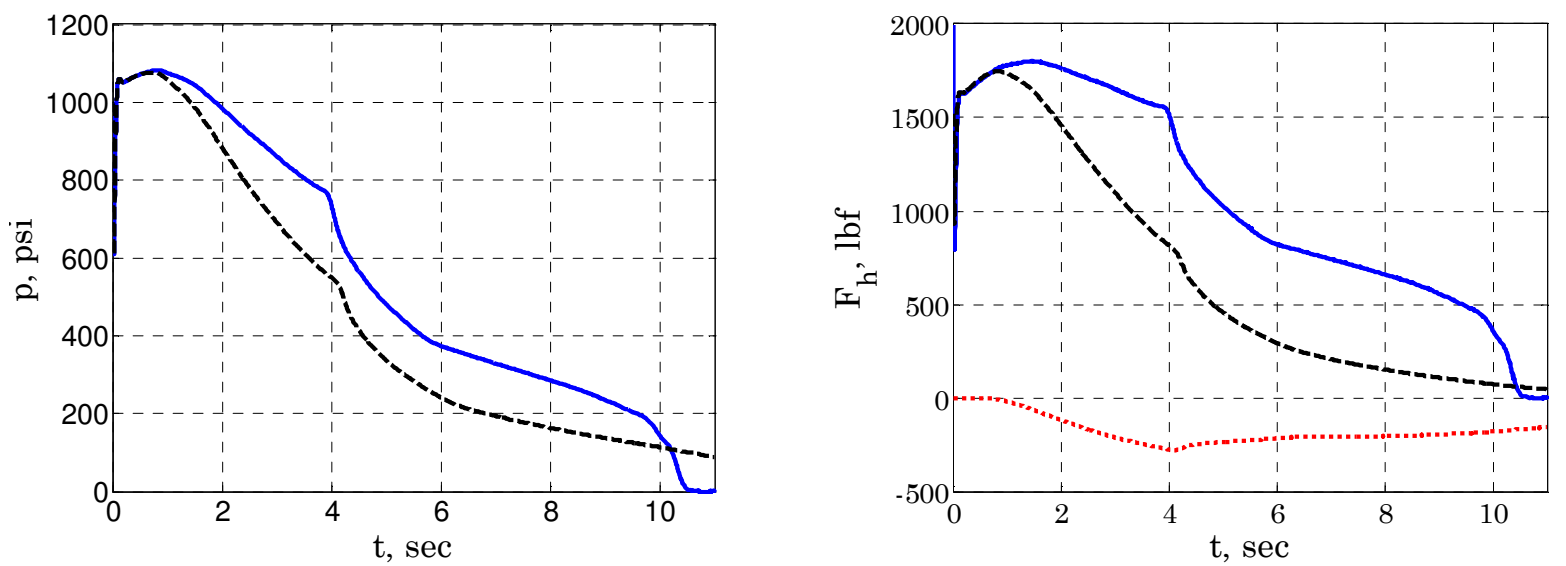

Figure 4 Typical time-series data generated by the LDPM (18). (left) Nominal pressure (blue line) as compared to the fault-induced pressure (solid dashed line). (right) Nominal thrust (blue line) as compared to the fault-induced thrust (dashed black line) and thrust generated by the hole (red dotted line). The fault occurs at $t f=0.5 \mathrm{sec}$. The erosion rate is approximately $0.15 \mathrm{in} / \mathrm{sec}$.

and to use the synthetic data generated by this model to verify the performance of the Bayesian algorithm as will be described in the next section.

\section{BAYESIAN INFERENTIAL FRAMEWORK FOR THE FD\&P OF THE CASE BREACH FAULT}

Note that effect of the case breach fault on the dynamics of the internal gas flow in SRBs is reduced to the effective modification of the nozzle throat area as explained above. It is, therefore, possible to infer SRB parameters using a Bayesian framework introduced in our earlier work ${ }^{10-13}$, for an analysis of the overpressure faults due to the changes of the nozzle throat area. In particular, it was shown ${ }^{13}$ that this algorithm can accommodate sudden changes of the model parameters and, therefore, is suitable for developing of the hybrid probabilistic IVHM of SRBs.

Here we briefly reproduce earlier results related to the analysis of the abrupt changes of the model parameters. The dynamics of the LDPM (18) can be in general be presented as an Euler approximation of the set of ODEs on a discrete time lattice $\left\{t_{k}=h k ; k=0,1, \ldots, K\right\}$ with time constant $h$

$$
x_{k+1}=x_{k}+h f\left(x_{k}^{*} \mid c\right)+\hat{\sigma} \sqrt{h} z_{k},
$$

where $z_{k}=\frac{1}{\sqrt{h}} \int_{t_{k}}^{t_{k}+h} \xi(t) d t, x_{k}^{*}=\frac{x_{k}+x_{k+1}}{2}, x_{k}=\left\{p, \rho, R, V, r_{h}, r_{t}, r_{i}\right\}$ is $L$-dimensional state of the system

(18), $\sigma$ is a diagonal noise matrix with two first non-zero elements $a_{1}$ and $a_{2}, f$ is a vector field representing the rhs of this system, and $c$ are parameters of the model. Given a Gaussian prior distribution for the unknown model parameters, we can apply our theory of Bayesian inference of dynamical systems ${ }^{10-13}$ to

$$
\begin{aligned}
& D_{i j}=\frac{h}{K} \sum_{k=0}^{K-1}\left(\dot{x}_{k}-f\left(x_{k} ; c\right)\right)_{i}\left(\dot{x}_{k}-f\left(x_{k} ; c\right)\right)_{j} \\
& c^{\prime}{ }_{l}=(A)_{m l}^{-1} w_{m},
\end{aligned}
$$

where elements $A_{m l}$ and $w_{m}$ are defined by the following equations

$$
w_{m}=h \sum_{k=0}^{K-1}\left[\sum_{n, n^{\prime}=1}^{L} U_{m n}^{\prime}\left(t_{k}\right) D_{n n^{\prime}}^{-1} \dot{x}_{n^{\prime}}\left(t_{k}\right)-\frac{\mathrm{v}_{m}}{2}\right]
$$




$$
A_{m l}=h \sum_{k=0}^{K-1}\left[\sum_{n, n^{\prime}=1}^{L} U_{m n}^{\prime}\left(t_{k}\right) D_{n n^{\prime}}^{-1} U_{n^{\prime} l}\left(t_{k}\right)\right] .
$$

Here the vector field is parameterized in special $\operatorname{form}^{10} f(x ; c)=\hat{U}(x) c$, where $U(x)$ is a block-matrix with elements $U_{m n}$ build of $N$ blocks of the form $\hat{I} \phi_{n}\left(x\left(t_{k}\right)\right), \hat{I}$ is $L x L$ unit matrix, and $\mathrm{v}_{m}(\mathbf{x})=\sum_{n=1}^{N} \frac{\partial U_{n m}(\mathbf{x})}{\partial x_{n}}$

To verify the performance of this algorithm for the diagnostics of the case breach fault we first assume the nominal regime of the SRB operation and check the accuracy and the time resolution with which parameters of the internal ballistics can be learned from the pressure signal only. To do so we notice that equations for the nozzle throat radius $r_{t}$, burn distance $R$, and combustion chamber volume can be integrated analytically for a measured time-traces of pressure and substituted into the equations for pressure dynamics. By noticing further that for small noise-intensities the ratio of dimensionless pressure and density $p / \rho \approx 1$ obtain the following equation for the pressure dynamics

$$
\dot{p}=-\frac{c_{0} \gamma \Gamma s_{t}}{V r_{b}} p+\frac{S_{b}}{V}\left(\gamma \rho_{p}-p\right) p^{n}+\sqrt{D} \xi_{2}(t),
$$

where $s_{t}(t), S_{b}(t)$, and $V(t)$ are known functions of time given by the following equations

$$
\begin{aligned}
& R(t)=\int_{0}^{t} p^{n}\left(t^{\prime}\right) d t^{\prime}, \quad S_{b}(t)=f(R(t)), \quad V(t)=V_{0}+\int_{0}^{R(t)} S_{b}(R) d R, \\
& s_{t}(t)=\pi r_{t}^{2}(t), \quad r_{t}(t)=\left[r_{t 0}^{1+\beta}+v_{m}(1+\beta) \int_{0}^{t} p^{1-\beta}\left(t^{\prime}\right) d t^{\prime}\right]^{\frac{1}{1+\beta}}
\end{aligned}
$$

The parameters $\frac{c_{0} \gamma \Gamma}{r_{b}} \gamma \rho_{p}$, and $D$ can now be inferred in the nominal regime by applying Eqs. (20)-(24) to the analysis of equation (25). An example of the inference results is shown in the Table $\mathbf{1 .}$

\begin{tabular}{c|ccc}
\multicolumn{1}{c}{ Parameter } & Actual & Inferred & Relative error \\
\hline$\gamma \rho_{p}$ & 248.2 & 244.7 & $1.4 \%$ \\
$-c 0 \Pi r_{b}$ & -61260 & -61347 & $1.38 \%$ \\
$\mathrm{D}$ & $2.5 \times 10^{-4}$ & $2.44 \times 10^{-4}$ & $2.4 \%$ \\
\hline
\end{tabular}

Table 1 The results of the parameter estimation of the model (18) in the nominal regime. The total time of the measurements in this test was $T=1 \mathrm{sec}$, the sampling rate was $1 \mathrm{kHz}$, and the number of measured points was $N=$ 1000.

We, therefore, conclude that the parameters of the nominal regime can be learned with good accuracy during the first second of the flight and can be assumed known in the further numerical tests of the Bayesian algorithm.

To extend these results to other types of faults we assume that the fault time evolution is in general a nonlinear function of time. We introduce the following general form of the nonlinear fault dynamics

$$
s_{h}(t)=a_{0}+a_{1} t+a_{2} t^{2}+a_{3} t^{3}+a_{4} t^{4}+a_{5} t^{5}
$$

We now use first few seconds of the measured time-series to infer fault parameters $\left\{a_{0}, a_{1}, a_{2}, a_{3}, a_{4}\right.$, $\left.a_{5}\right\}$. A few first seconds of the measured time series used to infer fault parameters are shown in the 

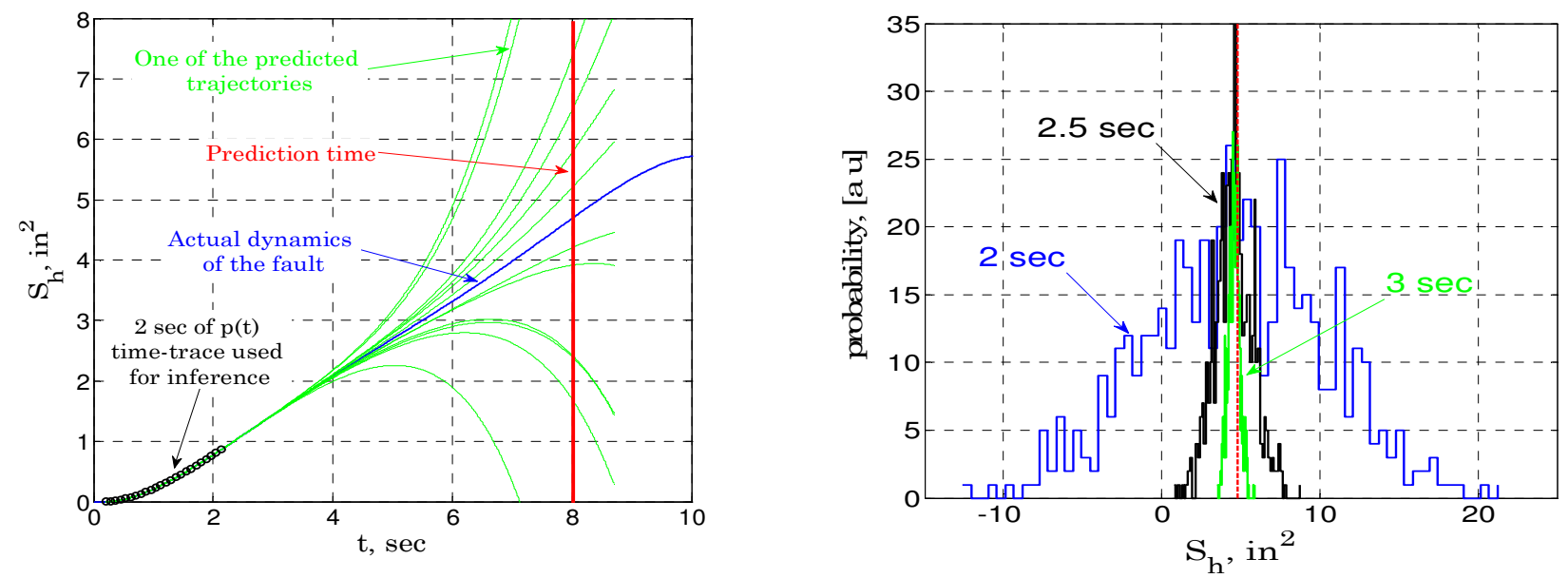

Figure 5 (left) Actual fault dynamics is shown by the blue solid line. The time interval elapsed from the case breach fault is shown by the black circles. The predicted trajectories of the fault are shown by thing green solid lines. The time moment of the prediction is indicated by a vertical red line. (right) The distribution (arbitrary units) of the predicted value of the fault at the future time $t=8 \mathrm{sec}$ is shown by solid blue, black, and green lines in comparison with the true value of the fault indicated by the vertical red dashed line. The time elapsed from the case breach fault used for predictions is shown in the figure.

Figure 5(left) by open circles. We assume known nominal parameters of the model and infer coefficients $a_{i}$ in the equation of the pressure dynamics, which in the presence of the case breach fault takes the form

$$
\dot{p}=-\frac{c_{0} \gamma \Gamma s_{t}}{V r_{b}} p-\frac{c_{0} \gamma \Gamma}{V r_{b}}\left(a_{0}+a_{1} t+a_{2} t^{2}+a_{3} t^{3}+a_{4} t^{4}+a_{5} t^{5}\right) p+\frac{S_{b}}{V}\left(\gamma \rho_{p}-p\right) p^{n}+\sqrt{D} \xi_{2}(t),
$$

Next we use the inferred values of the fault parameters to predict fault evolution forward in time. The predicted trajectories are shown in the Figure 5(left) by green lines in comparison with the actual time evolution shown by blue line. Next we build distributions of the predicted values of the hole area at a given future time (shown in the Figure 5(left) by the red vertical line). Next we investigate convergence of these distributions as a function of time elapsed from the onset of the fault. The distributions of the predicted values of the hole area at $8 \mathrm{sec}$ for three different instants of time at which predictions were made are shown in the Figure 5(right). It is clear from the figure that the accurate predictions can be made $5.5 \mathrm{sec}$ ahead in time using only $2.5 \mathrm{sec}$ of the time-traces to learn fault parameters.

\section{SUMMARY}

A detailed model of the case breach fault in a sub-scale motor has been developed. The model takes into account

- Real propellant geometry by using a key assumption the burn distance defines uniquely the burning area of the propellant;

- The ablation of the nozzle throat and the nozzle exit that includes the time evolution of roughness at the nozzle walls

- The fault dynamics that includes fault geometry and melting and burning of the hole walls in the metal case

- The time evolution of the volume of the combustion chamber

Under given assumptions the derived model can reproduce very accurately the time-traces of the internal SRB ballistics observed in the ground test firing. The derived LDPM is validated using FLUENT highfidelity model of the case breach fault. Using results of the numerical solution of the high-fidelity model 
in FLUENT we show that obtained LDPM can be used to find analytically a time evolution of the axial distributions of the gas flow parameters in the combustion chamber and in the nozzle.

The obtained LDPM is then incorporated into the Bayesian inferential framework as a part of the onboard FD\&P system for the next generation of NASA Heavy-Lift Launch Vehicles. It is shown that the obtained LDPM allows one to track in real time parameters of the SRB during the flight, to diagnose case breach fault, and to predict its values in the future times.

We note that a number of other SRB fault modes (including e.g. combustion instabilities, bore choking, and nozzle throat faults) can be reduced to the analysis of the time variation of the parameters of the SRB. In particular, the LDPM allows one to track in time changes in the burning area, volume of the combustion chamber, area of the effective nozzle throat, and thrust. Therefore, the approach developed in the present work method can be used to build FD\&P system for a variety of the fault modes in the SRB.

In conclusion we note that the derived LDPM can be used to analyze effectively the results of the ground and flight firing tests, to provide deep insight into the time variations of the SRB parameters in various nominal and off-nominal regimes, and to build a data base of dynamical signatures of various fault modes. The derived model can be further extended to the large scale motors taking into account that the zero-dimensional approximation is surprisingly accurate ${ }^{15}$ even when the nozzle stagnation pressure of large motor varies significantly in time, including the off-nominal regime of the case breach fault ${ }^{9}$.

\section{APPENDIX: THE ABLATION OF THE NOZZLE AND EROSION OF METAL}

The nozzle surface is heated by radiation $Q_{R}$ and convective $Q_{c}$ heat flows of combustion products (hot gas). The radius of the nozzle starts to erode when the surface temperature $T_{s}$ reaches an ablation temperature $T_{a b l}$. Let us estimate this time delay. The distribution of temperature at $r>r_{\mathrm{t}}$ is described by the equation

$$
C_{i n} \rho_{i n} \frac{\partial T}{\partial t}=\kappa_{i n}\left(\frac{\partial^{2} T}{\partial r^{2}}+\frac{1}{r} \frac{\partial T}{\partial r}\right)
$$

where $r$ is a coordinate normal to the surface (Figure 1, left). It is well known that the temperature distribution for $r>r_{\mathrm{t}}$ is characterized by the following thermo-diffusion length

$$
l_{D}=2 \sqrt{\kappa_{i n} t / C_{i n} \rho_{i n}}
$$

The heat flow $Q=Q_{c}+Q_{R}$ for the time interval $d t$ will heat the surface layer of an area $d S$ and a thickness of $l_{D}$. The heat balance equation can approximately be written as

$$
\left(Q_{c}+Q_{R}\right) d S d t=c_{m} \rho_{m} d S d\left[\left(T_{s}-T_{0}\right) l_{D}\right]
$$

where $T_{0}$ is an initial temperature of the nozzle. It follows from Eqs. (30) and (31)

$$
\frac{d T_{s}}{d t}+\frac{\left(T_{s}-T_{m 0}\right)}{2 t}=\frac{Q_{c}+Q_{R}}{2 \sqrt{C_{i n} \rho_{i n} \kappa_{i n} t}}
$$

Radiation heat flow is given by

$$
Q_{R}=\sigma\left[1-\exp \left(-\lambda p_{0}\right)\right]\left(T_{*}^{4}-T_{s}^{4}\right)
$$


where $\lambda=\left[0.001+4 \times 10^{-4} \times \% A L\right] / 14.69\left(\mathrm{in}^{2} / \mathrm{lb}\right)$ is the emissivity of the hot gas ${ }^{24}, \% A L$ is the percentage of aluminum in the solid propellant, and $\sigma=5.67 \times 10^{-8} \mathrm{~W} / \mathrm{m}^{2} \mathrm{~K}^{4}$ is the Stefan_Boltzmann constant [24]. The convective heat flow $Q_{c}=h_{g}\left(T-T_{s}\right)$ for $T_{s} \leq T_{a b l}$ where the heat transfer coefficient $h_{g}$ can be presented as [20,21]

$$
h=0.5 J C_{p} \eta_{r} C_{f}
$$

Here the coefficient $\eta_{r}$ describes an effect of the surface roughness. The value of coefficient $C_{f}$ is determined by Reynolds number $\operatorname{Re}=\rho u x / \mu$ which is about $10^{6}$ for typical parameters and high pressures. The critical Reynolds number which determines a transition between laminar and turbulent regimes is known to vary from approximately $10^{5}$ to $3 \times 10^{6}$, depending on the surface roughness and the turbulence level of the free stream [20, 21]. Thus, probably, the turbulence regime occurs even with a relatively low gas pressure due to growth of roughness of the nozzle surface. Therefore, coefficient $C_{f}$ can be approximated over a fairly wide range of Reynolds number by the Bartz's approximation [20-22]

$$
C_{f}=0.046\left(\frac{2 R_{n t} j}{\mu}\right)^{-0.2}\left(\frac{\mu C_{p}}{\kappa}\right)^{-0.67}=0.046 \operatorname{Re}^{-0.2} \operatorname{Pr}^{-0.67} .
$$

Here $\kappa$ is the thermal conductivity and $\mu$ is the dynamical viscosity of the hot gas, the Prandtl number $\operatorname{Pr}=\mu C_{p} / \kappa \simeq 1$ and the mass gas flow in the nozzle $j=\rho^{*} c^{*}=\left(\gamma p_{0} / \Gamma c_{0}\right)$. It follows from Eqs. (34)-(35)

$$
Q_{c}=0.023 \eta_{r} C_{p}\left(\frac{\gamma p_{0}}{\Gamma c_{0}}\right)^{0.8}\left(\frac{2 R_{n t}}{\mu}\right)^{-0.2}\left(T_{*}-T_{s}\right)
$$

where the gas temperature at the nozzle throat $T=T_{*}=2 T_{0} /(1+\gamma)$. The delay time $t_{d}$ is a moment when $T_{s}$ achieves the value of $T_{a b l}$. Its value is determined by Eqs. (32), (33) and (36). The estimations show that the delay time $t_{d}$ of heating of nozzle surface to the ablation temperature is very small, $t_{d}$ is much less than $0.1 \mathrm{sec}$.

The velocity of quasi-stationary propagation of the ablation front $v_{a b l}$ is determined by the equation of the heat balance for a small surface element $d S$ of the hole:

$$
\left(Q_{c}+Q_{R}\right) d S d t=\left[c_{i n}\left(T_{a b l}-T_{0}\right)+q_{i n}\right] \rho_{i n} v_{a b l} d t d S
$$

where $d t$ is a small time interval. It follows that

$$
\frac{d r_{t}}{d t}=v_{a b l}=\frac{Q_{c}+Q_{R}}{\left[c_{i n}\left(T_{a b l}-T_{0}\right)+q_{i n}\right] \rho_{i n}}
$$

This equation is valid for $\mathrm{t}>\mathrm{t}_{\mathrm{d}}$. We carried out numerical calculation and made sure that $Q_{c} \gg Q_{R}$ for the whole time period of interest to us. Taking into account this condition we find from Eqs. (36) and (38) that the ablation velocity can be written as 


$$
\frac{d r_{t}}{d t}=v_{a b l}=\eta_{r} v_{m}\left(\frac{p_{0}}{p_{\max }}\right)^{0.8}\left(\frac{r_{t}(t, x)}{r_{t 0}}\right)^{-0.2}
$$

where $r_{t 0}=r_{t}(0)$ and

$$
v_{m}=0.023 C_{p}\left(\frac{\gamma p_{\max }}{\Gamma c_{0}}\right)^{0.8}\left(\frac{2 R_{n t 0}}{\mu}\right)^{-0.2} \frac{\left(T_{*}-T_{a b l}\right)}{c_{i n}\left(T_{a b l}-T_{0}\right)+q_{i n}}
$$

We emphasized above that the coefficient $\eta_{r}$ describes an effect of the surface roughness: its value increases with the roughness [20-22]. To explain real data of nozzle ablation we introduce the approximation for this dependence given by Eq. [16] which describes well enough the experimental data.

The radius of the hole through a metal case starts to grow when the surface metal temperature $T_{s}$ reaches the melting or burning point. The time delay $t_{d m}$ is determined by an equation analogous Eq. (30). The velocity of propagation of the front of metal melting $v_{f}$ is determined at $t>t_{d m}$ by an equation analogous to Eq. (38):

$$
\frac{d r_{h}}{d t}=v_{f}=\frac{Q_{c}+Q_{R}}{\left[q_{m}+C_{m}\left(T_{m e l}-T_{m 0}\right)\right] \rho_{m}}
$$

Here $T_{m 0}$ is a temperature far from the hole surface and the radiation $Q_{R}$ and convective $Q_{c}$ heat flows are given by equations analogous to Eqs. (33) and (36):

$$
Q_{R}=\sigma\left[1-\exp \left(-\lambda p_{0}\right)\right]\left(T_{*}^{4}-T_{m e t}^{4}\right), \quad Q_{c}=0.023 \eta_{r} C_{p}\left(\frac{\gamma p_{0}}{\Gamma c_{0}}\right)^{0.8}\left(\frac{2 r_{h}}{\mu}\right)^{-0.2}\left(T_{*}-T_{m e t}\right)
$$

In addition the regression rate of the metal wall is governed by of erosion $v_{b}$. Experiments show that $v_{b} \simeq 0.3 \mathrm{in} / \mathrm{sec}$ for iron and carbon steels ${ }^{25}$. The same value of $v_{b}$ was found in a recent study by ATK Thiokol Inc. team, to explain the experimental data 9 . Thus, full velocity of increase of radius of a hole in the metal case is given by Eq. (14).

\section{REFERENCES}

1. F. E. C. Culick and V. Yang, "Prediction of the Stability of Unsteady Motions in Solid Propellant Rocket Motors," Nonsteady Burning and Combustion Stability of Solid Propellants, edited by L. De Luca, E. W. Price, and M. Summerfield, Vol. 143, Progress in Astronautics and Aeronautics, AIAA, Washington, DC, 1992, pp. 719-779.

2. F. E. C. Culick, "Combustion of the Stability in Propulsion Systems", Unsteady Combustion, Kluwer Academic Publisher, 1996.

3. G. A. Flandro et al., "Nonlinear Rocket Motor Stability Prediction: Limit Amplitude, Triggering, and Mean Pressure Shift", AIAA 2004-4054, 40th AIAA Joint Propulsion Conference \& Exhibit, July 2004, Flodira.

4. W. A. Dick et al. "Advanced Simulation of Solid Propellant Rockets from First Principles", Center for Simulation of Advanced Rockets, University of Illinois, 41st AIAA Joint Propulsion Conference \& exhibit, July 2005, Tucson, Arizona).

5. Isaac and M. P. Iverson, "Automated Fluid-Structure Interaction Analysis", ATK Thiokol Propulsion, A Division of ATK Aerospace Company, 2003. 
6. Q. Wang, B. W. Rex, R. P. Graham, "Fluid-structure interaction analyses of segmented soled rocket motors", ATK Thiokol, Inc., May, 2005.

7. V.V. Osipov, D.G. Luchinsky, V.N. Smelyanskiy, C. Kiris, D.A. Timucin, S.H. Lee, "In-Flight Failure Decision and Prognostic for the Solid Rocket Buster", AIAA-2007-5823, 43rd AIAA/ASME/SAE/ ASEE Joint Propulsion Conference and Exhibit, Cincinnati, OH, July 8-11, 2007.

8. Rogers Commission report (1986). Report of the Presidential Commission on the Space Shuttle Challenger Accident

9. J. E. McMillin, J. D. Leibold, M. E. Tobias, and N. J. Beach, "SRM Ballistic Failure Models for the $1^{\text {st }}$ tage Malfunction Turn Study Joint 1 failure and Case breach Model", Report TR015666 of ATK Thiokoi Inc., 2004.

10. V. N. Smelyanskiy, D. G. Luchinsky, D. A. Timuçin, and A. Bandrivskyy, "Reconstruction of stochastic nonlinear dynamical models from trajectory measurements", Physical Review E, Vol. 72, No. 2, 2005, 026202.

11. V. N. Smelyanskiy, D. G. Luchinsky, A. Stefanovska, and P. V. E. McClintock, "Inference of a Nonlinear Stochastic Model of the Cardiorespiratory Interaction", Phys. Rev. Lett. Vol. 94, No. 9, Mar. 2005, 098101.

12. V. V. Osipov, D. G. Luchinsky, V. N. Smelyanskiy, S. Lee, C. Kiris, D. Timucin, "Bayesian Framework for InFlight SRM Data Management and Decision Support", IEEE 2007 Aerospace Conference, Big Sky, March 2007.

13. D.G. Luchinsky, V.N. Smelyanskiy, V.V. Osipov, and D. A. Timucin, and S. Lee, "Data management and decision support for the in-flight SRM", AIAA-2007-2829 AIAA Infotech@Aerospace 2007 Conference and Exhibit, Rohnert Park, California, May 7-10, 2007.

14. V.N. Smelyanskiy, C. Kiris, V.V. Osipov, D.G. Luchinsky, D. Timucin, and S. Uckun, "Solid Rocket Motor Health Management for Safe Space Flight", paper AIAA-2006-6555, AIAA Guidance, Navigation, and Control Conference and Exhibit, Keystone, Colorado, Aug. 21-24, 2006.

15. M. Sailta, "Verification of Spatial and Temporal Pressure Distribution in Segmented Solid Rocket Motors," AIAA paper 89-0298, 27th Aerospace Science Meeting, Reno, Nevada, Jan. 9-12, 1989.

16. R. E. Sorkin, "Dynamics and Thermodynamics of Solid-Propellant Rockets", Wiener Bindery Ltd., Jerusalem, 1967.

17. L. D. Landay and E. M. Lifshits, Fluid Mechanics (Pergamon, 1987).

18. Salita, M., "Modern SRM ignition transient modeling. I - Introduction and physical models", AIAA-2001-3443, AIAA/ASME/SAE/ASEE Joint Propulsion Conference and Exhibit, 37th, Salt Lake City, UT, July 8-11, 2001

19. Salita, M., "Closed-Form Analytical Solutions for Fluid Mechanical, Thermochemical, and Thermal Processes in Solid Rocket Motors", AIAA 98-3965,34th Joint Propulsion Conference (Cleveland), 7/98.

20. F.P. Incropera and D. P. DeWitt, Introduction to Heat Transfer, John Wiley \& Sons, NY, 2002.

21. P. Hill and C. Peterson, Mechanics and Thermodynamics of Propulsion, 2-rd ed., Addison-Wesley Publishing Company, Inc. New York, 1992.

22. D. R. Bartz, Heat Transfer from Rapidly Accelerating Flow of Rocket Combustion Cases and from Heated Air, in Advances in Heat Transfer, vol. 2, Hartnett, J. P. , and Irvine, T. F. Jr., eds., New York: Academic Press, 1965.

23. R. M. Kendall, R. A. Rindal, and E. P. Bartlett, A Multicomponent Boundary Layer Chemically Coupled to an Ablating Surface, AIAA Journal, 5, 1063 (1967).

24. SPP technical documentation: "RocketWeb SPP Grain Design and Ballistics Inputs", Aththors: G. R. Nickerson, S. S. Dunn, and D. R. Berker, title: "The solid propellant rocket motor performance computer program (SPP), 1992.

25. P. L. Harrison and A. D. Yoffe, The Burning of Metals, Proceedings of the Royal Society of London, Series A, Math. and Physical Science, vol. 261, 357-370 (1961). 